\title{
Damage-based fracture with electro-magnetic coupling
}

\author{
P. Areias - H. G. Silva - N. Van Goethem • \\ M. Bezzeghoud
}

Received: 1 February 2012 / Accepted: 5 June 2012

(C) Springer-Verlag 2012

\begin{abstract}
A coupled elastic and electro-magnetic analysis is proposed including finite displacements and damage-based fracture. Piezo-electric terms are considered and resulting partial differential equations include a non-classical wave equation due to the specific constitutive law. The resulting wave equation is constrained and, in contrast with the traditional solutions of the decoupled classical electromagnetic wave equations, the constraint is directly included in the analysis. The absence of free current density allows the expression of the magnetic field rate as a function of the electric field and therefore, under specific circumstances, removal of the corresponding magnetic degrees-offreedom. A Lagrange multiplier field is introduced to exactly enforce the divergence constraint, forming a three-field variational formulation (required to include the wave constraint). No vector-potential is required or mentioned, eliminating the need for gauges. The classical boundary conditions of electromagnetism are specialized and a boundary condition involving the electric field is obtained. The spatial discretiza-
\end{abstract}

P. Areias $(\varangle) \cdot$ H. G. Silva $\cdot$ M. Bezzeghoud

Departamento de Física, Escola de Ciências e Tecnologia,

Universidade de Évora, Colégio Luís António Verney,

Rua Romão Ramalho, 59, 7002-554 Évora, Portugal

e-mail: pareias@civil.ist.utl.pt

URL: http://evunix.uevora.pt/ pmaa/

P. Areias

ICIST, Lisbon, Portugal

H. G. Silva · M. Bezzeghoud

CGE, Évora, Portugal

N. Van Goethem

Departamento de Matemática, Centro de Matemática

e Aplicacações Fundamentais, CMAF, Universidade de Lisboa,

Faculdade de Ciências, Av. Prof. Gama Pinto 2,

1649-003 Lisbon, Portugal tion makes use of mixed bubble-based (of the MINI type) finite elements with displacement, electric field and Lagrange multiplier degrees-of-freedom. Three verification examples are presented with very good qualitative conclusions and mesh-independence.

Keywords Electro-magnetism - Maxwell's equations · Elasticity $\cdot$ Piezo-electricity $\cdot$ Mixed finite element methods

\section{Introduction}

Fundamental theoretical contributions for the analysis of continuum elastic dielectrics were made by Lax and Nelson [1] and Maugin [2]. The latter text contains contributions with a detail beyond what is intended with this work. The purpose of it is to inaugurate a specific approach to electromagnetic and elasticity coupling implemented with mixed finite elements to deal with fracture (with finite strains). Piezo-electricity is also included, but mainly as a constitutive ingredient. Recent discussions on this topic, also with a continuum approach, are the works of Ericksen (cf. [3,4]) and Dorfmann and Ogden [5] albeit limited to strict dielectrics (without the magnetic field). Numerical calculations are sparse and mainly limited to magnetostatics (see, for example [6]) and piezo-electricity. Kuna [7] performed a theoretical and experimental review of piezo-electricity with classical fracture mechanics. In [8], the same author used a electromechanical contour integral to calculate energy release rates.

We perform finite displacements and rotations but small strains (in the sense of Bathe [9] p. 595 paragraph 6.6.3) in order to account for the (possibly large) rotation of the crack faces. The extension to finite strains demands an in-depth study of the piezo-electric term in the total stress, beyond of the scope of this paper. Simulations of electro-magnetic 
fields including piezo-electricity are performed. The total stress contains the Cauchy stress, the Maxwell stress, the piezo-electric stress and the polarization stress. The piezoelectric effect is characterized as a coupling between the electric polarization of a given material and the (mechanical) stress field. It is crucial in sensors, actuators, smart materials among others. There is a significant variety of piezo-electric materials: crystals, e.g. quartz; ceramics, e.g. lead zirconate titanate (PZT); polymers, e.g. polyvinylidene fluoride.

Taking into account that these materials are subjected to frequent mechanical actions, it is of fundamental importance the study of fracture, in order to estimate their reliability to device applications. For that reason, many works have been focused on this topic, for a recent review the reader is referred to [7]. Interestingly, the majority of them only consider the electrostatic limit where the coupling of the electric and magnetic fields (present in Maxwell's equations) can be neglected and the analysis can be restricted to the electric field. Obviously, this constitutes an overall simplification to the problem since in this case the electric field (vector), $\boldsymbol{e}$, can be calculated from the electric potential, $\phi$, reducing a $n$-dimensional problem (2 or 3, typically) to a one-dimensional problem.

The basic equations considered in this paper are classical (Maxwell, piezoelectric polarization, etc) but their coupling with fracture propagation has not been studied much so far. So, the contributions of this paper are the following:

- Numerical simulation of fracture incorporating magnetic and electric fields taking into account piezoelectric effects.

- A stable mixed formulation with Lagrange multipliers for imposing the null divergence of the electric displacement.

- Non-standard formulation resulting in a uncoupled wave equation.

The remaining of this paper is composed of six sections. Section 2 contains the basic premises of our work, as well as original derivations for the weak form and boundary conditions. Section 5 briefly summarizes the discretization proposal and the mixed finite element. Section 3 presents the relevant constitutive law with a piezo-electric term and the proof of its frame-invariance. Section 2 describes the mixed finite element technique and presents the final discretized equations. Three numerical applications are described in Sect. 6 where the new technique is successfully tested and, finally, in Sect. 7 some conclusions are drawn.

\section{Maxwell and equilibrium equations}

We consider a linear elastic material with a damage evolution law, equilibrium with moderate strains and finite dis- placements (ensuring the validity of Hooke's law) and the classical electrodynamics of continua (cf. [1,2]).

The considered governing equations consist of:

- Cauchy equations of motion using the electromagnetic force as the only volume force.

- Mass conservation.

- Maxwell's equations in S.I. units for dielectrics, in the absence of free charges and currents.

- Linear constitutive equations of electromagnetism, stressstrain relations and piezo-electricity.

Figure 1 shows the idealization of the typical problem to be solved and the three types of boundary conditions (essential for the displacement unknown and electric field and natural boundary conditions).

The mass conservation principle for an impermeable continuum (open set $\Omega$ ) is concisely given by:

$\frac{\partial(J \rho)}{\partial t}=0$ in $\Omega$

where $\rho$ is the spatial mass density and $J=\operatorname{det} \boldsymbol{F}$ where $\boldsymbol{F}$ is the deformation gradient (cf. [10]).

The Cauchy equation of motion and Cauchy Lemma read:

$\nabla \cdot \sigma^{T}+f=\rho \ddot{\boldsymbol{u}}$ in $\Omega$

$\boldsymbol{\sigma} \boldsymbol{n}=\overline{\boldsymbol{t}}$ in $\Gamma_{t}$

where $\boldsymbol{\sigma}$ is the Cauchy stress tensor, $\boldsymbol{u}$ is the displacement vector and $t \in[0, T]$ is the time variable (not to be mistaken with $\bar{t}$, the prescribed stress vector) and the term $\boldsymbol{f}$ is the body force field. Note that, according to our notation, $\sigma_{i j}$ is the Cauchy stress component at facet $j$ with the direction $i$. This is of course standard (e.g. $[10,11])$ and shown here for completeness. A useful notion in elastic dielectricity is the one of total stress, here denoted as $\sigma^{\star}$ which allows the rewriting of (2) as:

$\nabla \cdot \sigma^{\star^{T}}=\rho \ddot{u}$

We consider $\rho \ddot{\boldsymbol{u}}=\mathbf{0}$ in remaining of this work. Maxwell's equations in classical form ${ }^{1}$ in a domain $\Omega$ (cf. [4,12-14]) are concisely written as:

$\boldsymbol{\nabla} \times \boldsymbol{e}+\dot{\boldsymbol{b}}=\mathbf{0}$ in $\Omega$

$\nabla \cdot \boldsymbol{b}=0$ in $\Omega$

$\nabla \times \boldsymbol{h}-\dot{\boldsymbol{d}}=\boldsymbol{J}_{f}$ in $\Omega$

$\boldsymbol{\nabla} \cdot \boldsymbol{d}=q_{f}$ in $\Omega$

where $\boldsymbol{e}$ is the electric field, $\boldsymbol{b}$ is the vector of magnetic induction, $\boldsymbol{h}$ is the magnetic field and $\boldsymbol{d}$ is the electric displacement current (see, e.g. [2,12]). The terms in the right-hand sides of (7) and (8) are the free external current $\left(q_{f}\right)$ and charge

\footnotetext{
${ }^{1}$ S.I. units are adopted and lower-case is used for spatial quantities.
} 
Fig. 1 Relevant ingredients for the coupled equilibrium/electromagnetic problem (current configuration). The electric field equation holds in the interior and in the exterior of $\Omega$ with matching of the external and internal values on every portion of the boundary where $q_{s}=0$

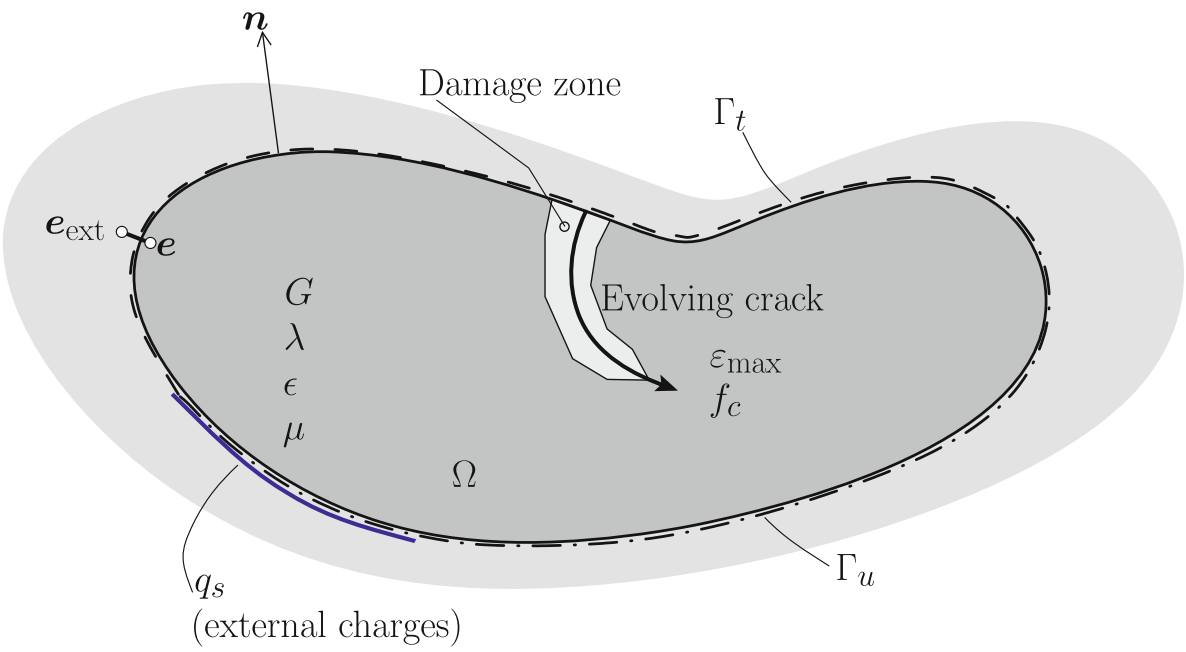

$\left(\boldsymbol{J}_{f}\right)$, respectively, and are related by the continuity equation: $\dot{q}_{f}+\boldsymbol{\nabla} \cdot \boldsymbol{J}_{f}=0$. It is noticeable that the validity of (5-8) for a continuum described in the spatial configuration is shown by Lax and Nelson [1]. Here, the following notation is adopted (see also [1]):

$\dot{\bullet}=\frac{\partial \bullet}{\partial \mathrm{t}}$

The inclusion of piezo-electricity in the system (5-8) can made by means of the polarization vector or directly by introduction in a constitutive law for $\boldsymbol{d}$. The latter is used here. As a matter of fact, for piezo-electric materials under consideration, we introduce a linear relation between the electric displacement, the electric field and the Almansi strain $\boldsymbol{\varepsilon}$ :

$\boldsymbol{d}=\epsilon \boldsymbol{e}+\mathscr{A}: \boldsymbol{\varepsilon}$

where $\epsilon$ is the electric permitivity ${ }^{2}$ and $\mathscr{A}$ is a general thirdorder tensor. The Almansi strain is defined in terms of the deformation gradient $\boldsymbol{F}$ by:

$\boldsymbol{\varepsilon}=\frac{1}{2}\left(\boldsymbol{I}-\boldsymbol{F}^{-T} \boldsymbol{F}^{-1}\right)$

The choice of this strain is justified by the fact that, in contrast with the small strain measure (the symmetric gradient of the displacement), allows large rotations of the crack faces without spurious stress.

We specialize the general relation (10) by introducing the third-order piezo-electric tensor $\mathscr{J}$ :

$\mathscr{A}=-\epsilon \mathscr{I}$

The tensor $\mathscr{I}$ is minor-symmetric in the two last indices, i.e. $[\mathscr{I}]_{i j k}=[\mathscr{I}]_{i k j}$. Relating the continuum and vacuum permitivity $\epsilon=(1+\chi) \epsilon_{0}$ with $\chi$ being the electric susceptibility we obtain the polarization as a constitutive relation:

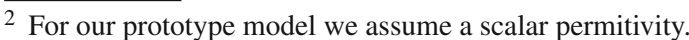

$\boldsymbol{p}=\epsilon_{0} \chi \boldsymbol{e}-\epsilon \underbrace{\mathscr{I}: \boldsymbol{\varepsilon}}_{\boldsymbol{q}}$

where $\epsilon_{0} \chi \boldsymbol{e}$ is the average electric dipole moment density and $\epsilon \mathscr{I}: \varepsilon$ is the piezo-electric polarization term. For notation simplicity, the electric displacement (10) is rewritten as:

$\boldsymbol{d}=\epsilon(\boldsymbol{e}-\boldsymbol{q})$

Note that $\boldsymbol{q}$ is unrelated to the electric charge. Our formulation is a particular case of Kuna [8] where we restrict to one dielectric constant. In homogeneous linear isotropic continua, for which Lorentz relations hold, we can write (7) and (8) as:

$\nabla \times \boldsymbol{b}=\mu \boldsymbol{J}_{f}+\mu \epsilon(\dot{\boldsymbol{e}}-\mathscr{I}: \dot{\boldsymbol{\varepsilon}})$ in $\Omega$

$\epsilon \boldsymbol{\nabla} \cdot(\boldsymbol{e}-\mathscr{I}: \boldsymbol{\varepsilon})=q_{f}$ in $\Omega$

where $\mu$ is the magnetic permeability. Both $\epsilon$ and $\mu$ can be related to the corresponding vacuum constants. A direct manipulation results in the following second-order system:

$$
\begin{aligned}
& \nabla \times(\nabla \times \boldsymbol{e})+\mu \dot{\boldsymbol{J}}_{f}+\mu \epsilon(\ddot{\boldsymbol{e}}-\ddot{\boldsymbol{q}})=\mathbf{0} \\
& \nabla \cdot(\boldsymbol{e}-\boldsymbol{q})=\frac{q_{f}}{\epsilon}
\end{aligned}
$$

It is clear that $\nabla \cdot \boldsymbol{b}=0$ is trivially satisfied in $[0, T]$ provided $(\nabla \cdot b)(0)=\mathbf{0}$ is satisfied. Moreover, the reader can verify that, contrary to traditional derivations in electromagnetic wave propagation, no Laplacian of $\boldsymbol{e}$ emerges in (17) since the classical condition $\nabla \cdot \boldsymbol{e}=0$ does not hold, due to the piezo-electric term in (18). An additional note is required: in the notation of Bustamante and Ogden [15], the polarization is determined differently. After direct specialization of the general boundary conditions (cf. [1]), a final set of boundary conditions for piezo-electricity and electromagnetic coupling is obtained (see also $[15,16]$ for a comprehensive description): 
$\left[\left[\frac{1}{\mu} \boldsymbol{b}\right]\right] \times \boldsymbol{n}=\boldsymbol{J}_{s}$ in $\Gamma$

$[[\boldsymbol{e}]] \times \boldsymbol{n}=\mathbf{0}$ in $\Gamma$

$[[\epsilon(\boldsymbol{e}-\boldsymbol{q})]] \cdot \boldsymbol{n}=q_{s}$ in $\Gamma$

$[[\boldsymbol{b}]] \cdot \boldsymbol{n}=0$ in $\Gamma$

We use the notation $[[\bullet]]$ for the "jump" of $\bullet$ : the difference between the external and internal values of $\bullet$ (see Fig. 1). It can be proven that the condition $[[\boldsymbol{b}]] \cdot \boldsymbol{n}=0$ is trivially satisfied. Contrary to the charge surface density, $q_{s}$, the surface current density $\boldsymbol{J}_{s}$ is not directly imposed in this framework (see also the recent paper by Linder et al. [17]). Since the magnetization vector is here considered null, we can use the conclusions in reference [18] to write the enforced boundary conditions:

$[[\boldsymbol{b}]]=\mathbf{0}$ in $\Gamma$

$\boldsymbol{e}=-\frac{q_{s}}{\epsilon} \boldsymbol{n}$ in $\Gamma$

For two-dimensional problems (23-24) results from (19-22) in the absence of surface current density. Note that the complete $\boldsymbol{e}$ is specified at $\Gamma$, in contrast with what occurs in piezo-electric reports. Typically (see [17]) the unknown field is $\boldsymbol{d}$ and not $\boldsymbol{e}$ since the magnetic field is seldom included in numerical simulations. For dielectrics (considered in this work) it follows that $\boldsymbol{J}_{f}=\mathbf{0}$ and $q_{f}=0$ (see, for example, the book of Maugin [2] p. 157).

\section{Coupled constitutive law}

\subsection{Total stress}

We make use of the compressible Neo-Hookean hyperelastic model (cf. Eq. (6.29) of [19]) as a function of the deformation gradient $\boldsymbol{F}$. The constitutive law for the total stress is given as $^{3}$ :

$$
\begin{aligned}
\boldsymbol{\sigma}^{\star}= & \underbrace{(1-f)\left[\frac{G}{J}\left(\boldsymbol{F} \boldsymbol{F}^{T}-\boldsymbol{I}\right)+\frac{\lambda}{J} \ln J \boldsymbol{I}\right]}_{\boldsymbol{\sigma}_{e}} \\
& +\underbrace{\frac{1}{\mu} \boldsymbol{b} \otimes \boldsymbol{b}+\epsilon \boldsymbol{e} \otimes \boldsymbol{e}-\frac{1}{2}\left(\epsilon\|\boldsymbol{e}\|^{2}+\frac{1}{\mu}\|\boldsymbol{b}\|^{2}\right) \boldsymbol{I}}_{\boldsymbol{\sigma}_{M}} \\
& +\underbrace{\epsilon \boldsymbol{e} \cdot \mathscr{I}}_{\boldsymbol{\sigma}_{P E}}
\end{aligned}
$$

where $\sigma_{e}$ is the elastic stress, $\sigma_{M}$ is the Maxwell stress and $\sigma_{P E}$ is the piezo-electric stress. Note that the electric field

\footnotetext{
3 In contrast with Chapter 4 of [2], we do not include electro-magnetic terms in the body forces.
}

already accounts for the piezo-electric effect in the global PDE in (17) and hence the reciprocal piezo-electric law is enforced at the global level. It is noticeable that the polarization stress (see [2]) is not present in (25) for thermodynamically consistent Cauchy stresses (see [20] for this term and also [15] for a similar expression). Note that the transfer of body forces to the total stress has been an implicit practice in the literature (cf. Vu, Steinmann and Possart [20] Eqs. (12) and (13)). It is considered as standard (cf. [21], Chap. 7) that in (25), the elastic stress shows a term with the factor $(1-f)$ where the void fraction $f$ is a constitutive variable accounting for material softening. ${ }^{4}$ We use a phenomenological model (see also [22]) which is sufficient for this work's purpose - the so-called damage variable in Lemaitre's work is here denoted void fraction. Also included in (25) is the third order piezo-electric tensor $\mathscr{I}$. We must remark that this damage model is unsuited for elastomer damage representation in the sense of Mullins [23]. In contrast with the works of Bustamante and Ogden in electroelasticity (see [15]) and magnetoelasticity (see [16]), two differences are worth mentioning:

- The aforementioned authors consider incompressible materials.

- We limit the analysis to finite displacements and small strains (in the sense of Bathe [9] p. 595 paragraph 6.6.3).

\subsection{Damage representation}

Damage modeling includes a loading function and the loading/unloading conditions. The corresponding void fraction loading function is:

$$
\begin{aligned}
\varphi(\boldsymbol{\varepsilon})= & {\left[\frac{\varepsilon_{\max }}{\varepsilon_{1}}\left(1+\alpha \frac{\varepsilon_{1}}{\varepsilon_{\max }}-\alpha\right)\right] } \\
& \times \exp \left[\alpha\left(1-\frac{\varepsilon_{1}}{\varepsilon_{\max }}\right)-1\right]-(1-f),
\end{aligned}
$$

where $\varepsilon_{1}$ is the maximum principal strain and $\varepsilon_{\max }$ is the maximum principal strain attainable during the loading history at a given point before softening. Definition (26) precludes $f$ of attaining 1 for finite $\varepsilon_{1}$ since $\varphi(\boldsymbol{\varepsilon}) \leq 0$. The parameter $\alpha$ in (26) is given as:

$\alpha= \begin{cases}1 & \varepsilon_{1} \leq \varepsilon_{\max } \\ \frac{l}{l_{c}} & \varepsilon_{1}>\varepsilon_{\max }\end{cases}$

where the parameter $l$ is the mesh size length and the $l_{c}$ is a characteristic length scale (cf. Oliver [24]). Figure 2 shows a representation of this prototype model. It is simple to prove that the softening energy is of course dependent on the length scale using $\alpha$ as calculated in (27).

\footnotetext{
${ }^{4}$ In the sense of Lemaitre [22], valid for small strains.
} 


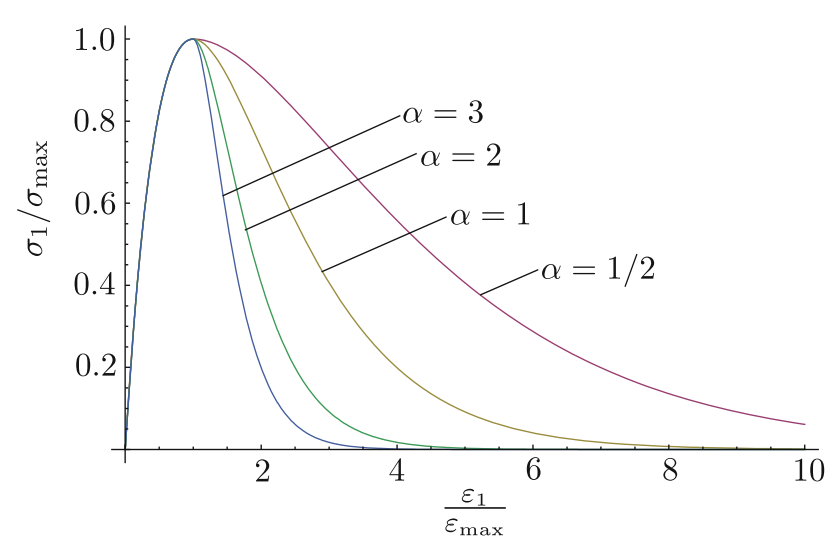

Fig. 2 One-dimensional representation of the damaged stress/strain relation

For a related Rankine linear damage law see Lemaitre and Chaboche [21], p. 363. The following loading/unloading conditions are used for our Rankine-type model:

$\varphi(\boldsymbol{\varepsilon}) \leq 0$

$\dot{f} \varphi(\boldsymbol{\varepsilon})=0$

$\dot{f} \geq 0$

and correspond to the standard approach to damage.

\subsection{Piezo-electric matrix and orientation of axes}

The coupling between mechanical and electric fields depends on the orientation of a piezo-electric crystal. The invariance of the scalar $\boldsymbol{d} \cdot(\mathscr{I}: \boldsymbol{\varepsilon})$ (the reader can verify that this expression has units of energy) allows the writing of the following transformation of the piezo-electric matrix as a third-order tensor:

$$
[\mathscr{I}]_{l m n}=[\boldsymbol{Q}]_{l i}[\boldsymbol{Q}]_{m j}[\boldsymbol{Q}]_{n k}[\widetilde{\mathscr{I}}]_{i j k}
$$

where the elements of $\boldsymbol{Q}$ are obtained from two orthonormal basis $\boldsymbol{e}_{j}$ and $\widetilde{\boldsymbol{e}}_{i}$ :

$$
[\boldsymbol{Q}]_{i j}=\widetilde{\boldsymbol{e}}_{i} \cdot \boldsymbol{e}_{k} R_{k j}=\cos \alpha_{i k} R_{k j}
$$

where $\alpha_{i j}$ are the internal angles between the basis vectors and $\boldsymbol{R}$ is the rotation matrix, obtained from the polar decomposition. It is obvious that minor-symmetry in the last two indices of $[\mathscr{I}]$ allows some computational savings in (31). It is here assumed that the tabulated piezo-electric properties correspond to the tensor components $[\widetilde{\mathscr{I}}]$ and $\alpha_{i j}$ are problem-dependent.

\subsection{Frame invariance}

Equation (25) is frame-invariant in the sense that [25, pp. 41-43]:

$\boldsymbol{x}^{\prime}=\boldsymbol{c}(t)+\boldsymbol{Q}(t) \boldsymbol{x} \Rightarrow \boldsymbol{\sigma}^{\star^{\prime}}=\boldsymbol{Q}(t) \boldsymbol{\sigma}^{\star} \boldsymbol{Q}(t)^{T}$

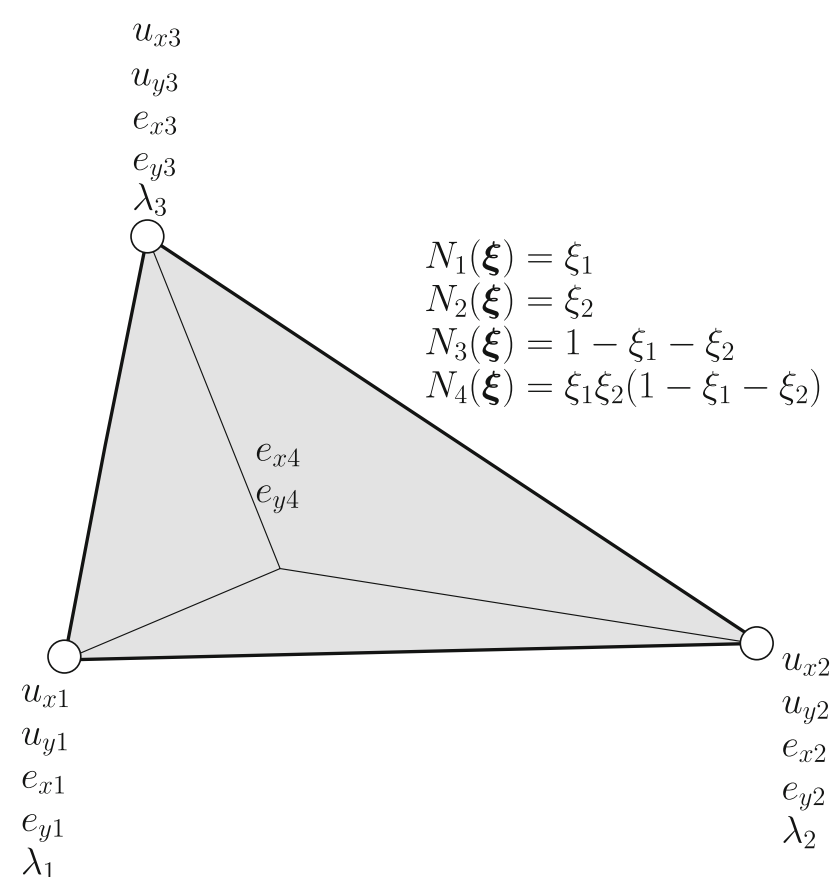

Fig. 3 Use of MINI element ([29]) for the mixed $\boldsymbol{u}-\boldsymbol{e}-\boldsymbol{\lambda}$ problem

where $\boldsymbol{c}(t)$ is a point and $\boldsymbol{Q}(t)$ is a unimodular orthogonal tensor. In Eq. (25), the first term $\left(\sigma_{e}\right)$ is trivially frame-invariant since $J^{\prime}=J, f^{\prime}=f, \boldsymbol{I}=\boldsymbol{Q} \boldsymbol{Q}^{T}$ and $\boldsymbol{F}^{\prime} \boldsymbol{F}^{\prime T}=\boldsymbol{Q} \boldsymbol{F} \boldsymbol{F}^{\boldsymbol{T}} \boldsymbol{Q}^{T}$ (with $t$ omitted). The tensor products $\boldsymbol{b} \otimes \boldsymbol{b}=\boldsymbol{b} \boldsymbol{b}^{T}$ and $\boldsymbol{e} \otimes \boldsymbol{e}=\boldsymbol{e} \boldsymbol{e}^{T}$ are also frame-invariant. ${ }^{5}$ The norms of $\boldsymbol{e}$ and $\boldsymbol{b}$ are frame-invariant from orthogonality of $\boldsymbol{Q}$. Finally, the only term that may raise doubt is $\sigma_{P E}$ which, from the definitions (32) and (31) can be proved to be frame-invariant.

\section{Governing equations in reduced wave form}

After the introduction of the previous derivations and considering a two-dimensional problem, the final equations to integrate are therefore dependent on $\sigma^{\star}$ and independent of b:

$$
\begin{aligned}
& c^{2}[\nabla \times(\nabla \times \boldsymbol{e})]+(\ddot{\boldsymbol{e}}-\ddot{\boldsymbol{q}})=\mathbf{0} \text { in } \Omega \\
& \nabla \cdot \boldsymbol{\sigma}^{\star T}=\mathbf{0} \text { in } \Omega \\
& \nabla \cdot(\boldsymbol{e}-\boldsymbol{q})=0 \text { in } \Omega \\
& {[[\nabla \times \boldsymbol{e}]]=\mathbf{0} \text { in } \Gamma} \\
& \boldsymbol{e}=-\frac{q_{s}}{\epsilon} \boldsymbol{n} \text { in } \Gamma \\
& \boldsymbol{\sigma} \boldsymbol{n}=\overline{\boldsymbol{t}} \text { in } \Gamma_{t} \\
& \boldsymbol{u}=\overline{\boldsymbol{u}} \text { in } \Gamma_{u}
\end{aligned}
$$

\footnotetext{
${ }_{5}$ In the sense that material fields are obtained as $\boldsymbol{E}=\boldsymbol{F}^{T} \boldsymbol{e}$ and $\boldsymbol{B}=$ $\boldsymbol{F}^{T} \boldsymbol{b}$.
} 
with $c^{2}=1 /(\mu \epsilon)$ being the square of the light velocity in the continuum. Note that no explicit body forces appear since the electro-magnetic field effect on the forces is completely included in $\sigma^{\star}$ [in Eq. (25)] and the Cauchy lemma retains its original form [in Eq. 39]. The weak form of the above equations is obtained by using a Lagrange multiplier field, $\lambda \equiv \lambda(\boldsymbol{x})$ and performing the customary projections and use of Green's theorem. We introduce the spaces for the test functions $\mathscr{V}_{u}=\left\{\delta u_{i} \in H^{1}(\Omega) \mid \delta \boldsymbol{u}=\right.$ $\mathbf{0}$ in $\left.\Gamma_{u}\right\}, \mathscr{V}_{d}=\left\{\delta d_{i} \in H^{1}(\Omega) \mid \delta \boldsymbol{d}=\mathbf{0}\right.$ in $\left.\Gamma\right\}$ and $\mathscr{P}=\left\{\delta \lambda \in L^{2}(\Omega) \mid \delta \lambda=0\right.$ in $\left.\Gamma\right\}$ and the sets for the trial functions $\mathscr{D}_{u}=\left\{u_{i} \in H^{1}(\Omega) \mid u_{i}=\bar{u}_{i} \quad\right.$ in $\left.\quad \Gamma_{u}\right\}, \mathscr{D}_{d}=$ $\left\{e_{i} \in H^{1}(\Omega) \mid e=\bar{e}_{i}\right.$ in $\left.\Gamma\right\}$ and $\mathscr{P}$ (the Lagrange multiplier space for test functions and set for trial functions coincide). The problem statement in weak form reads: find

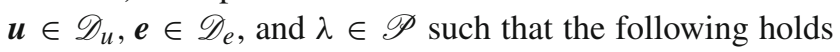
(see [26] for this nomenclature and symbols):

$$
\int_{\Omega} c^{2}(\nabla \times \boldsymbol{e}) \cdot(\nabla \times \delta \boldsymbol{d}) \mathrm{d} \Omega+\int_{\Gamma} c^{2} \underbrace{[(\nabla \times \boldsymbol{e}) \times \delta \boldsymbol{d}] \cdot \boldsymbol{n}}_{-[(\nabla \times \boldsymbol{e}) \times \boldsymbol{n}] \cdot \delta \boldsymbol{d}=0} \mathrm{~d} \Gamma
$$

$$
\begin{aligned}
& +\int_{\Omega}(\ddot{\boldsymbol{e}}-\ddot{\boldsymbol{q}}) \cdot \delta \boldsymbol{d} \mathrm{d} \Omega+\int_{\Omega} \boldsymbol{\sigma}: \nabla^{s} \delta \boldsymbol{u} \mathrm{d} \Omega-\int_{\Gamma_{t}} \overline{\boldsymbol{t}} \cdot \delta \boldsymbol{u} \mathrm{d} \Gamma_{t} \\
& +\int_{\Omega} \lambda \delta[\nabla \cdot(\boldsymbol{e}-\boldsymbol{q})] \mathrm{d} \Omega+\int_{\Omega} \delta \lambda[\nabla \cdot(\boldsymbol{e}-\boldsymbol{q})] \mathrm{d} \Omega=0
\end{aligned}
$$

$\forall \delta \boldsymbol{d} \in \mathscr{V}_{d}, \forall \delta \boldsymbol{u} \in \mathscr{V}_{u}, \forall \delta \lambda \in \mathscr{P}$. The test functions $\delta \boldsymbol{d}, \delta \boldsymbol{u}$ and $\delta \lambda$ in (41) can be seen as variations of $\boldsymbol{d}, \boldsymbol{u}$ and $\lambda$, respectively. We make use of the Acegen add-on [27] to the Mathematica software [28] to accomplish the derivation of the discretized equilibrium equations and corresponding linearization. The explicit expressions are omitted in this report. It is also noticeable that, in (41), the symmetric spatial gradient of $\delta \boldsymbol{u}$ is adopted: $\nabla^{s} \delta \boldsymbol{u}=\left[\nabla \delta \boldsymbol{u}+(\nabla \delta \boldsymbol{u})^{T}\right] / 2$. Initial conditions for (41) are given for both $\boldsymbol{u}$ and $\boldsymbol{e}$ (taken as homogeneous in this work). A remark concerning the conditioning of (41) is in order: for a PZT material, with $\mu=1.256 \times 10^{-6} \mathrm{Cm}^{-2}, \epsilon=6 \times 10^{-9} \mathrm{Fm}^{-1}, E=$ $69.59 \times 10^{9} \mathrm{Nm}^{-2}, v=0.357$ and $\rho=7500 \mathrm{Kg} \mathrm{m}^{-3}$, the
Fig. 4 PZT specimen with relevant properties and dimensions. The (artificially coarse) mesh is shown for illustrative purposes only
Fig. 5 Displacement/time results for point $A$ for two mesh densities
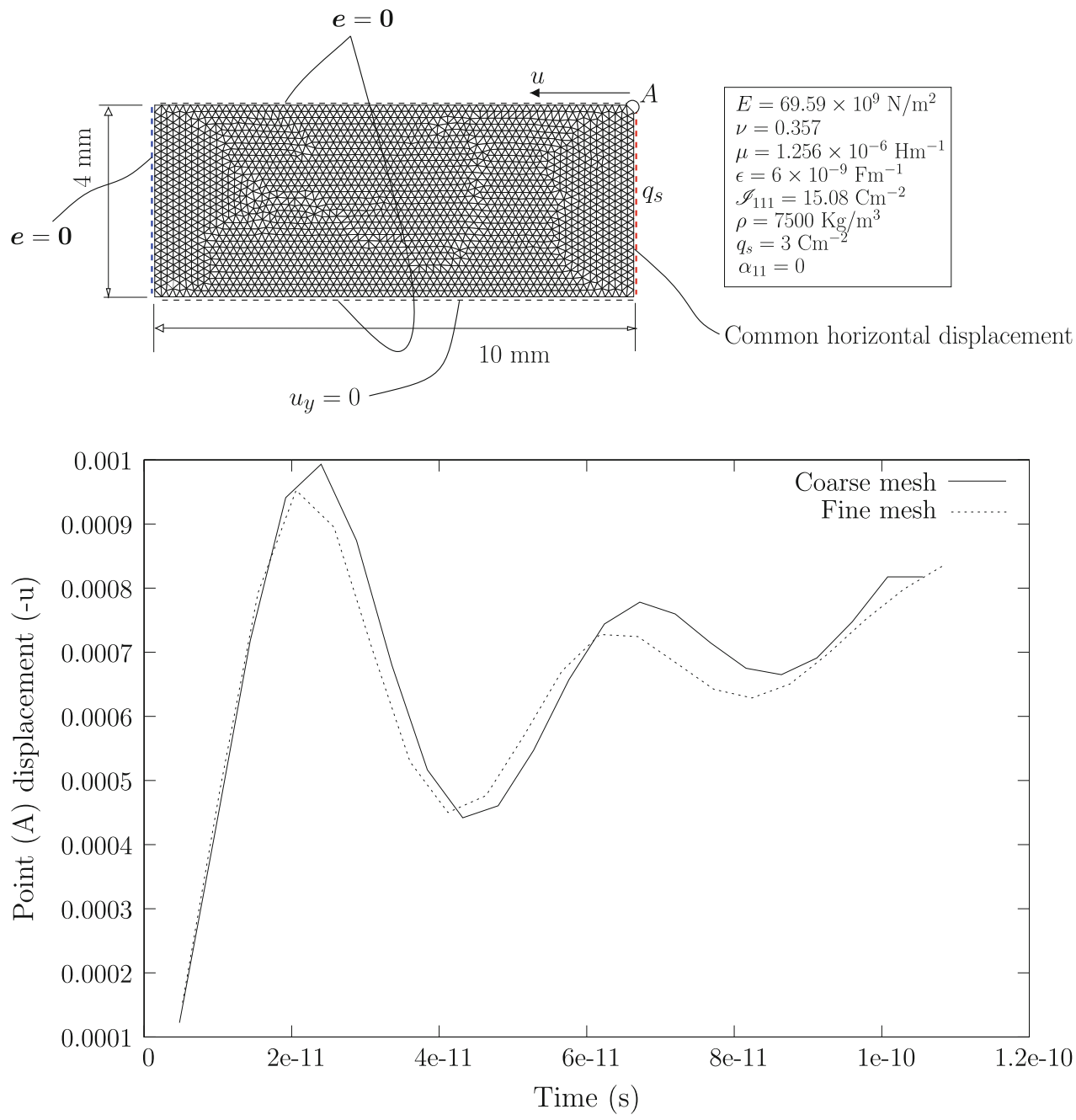
(a)

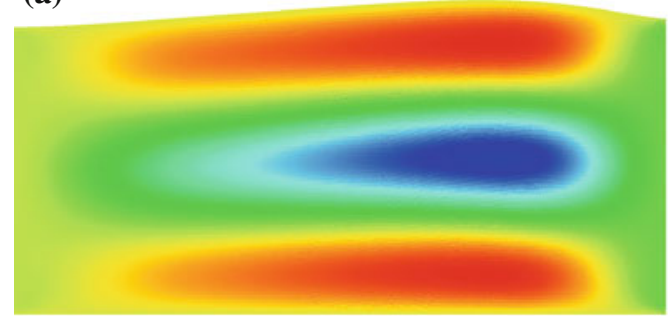

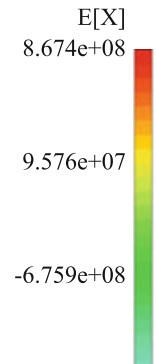

$-1.448 \mathrm{e}+09$

$-2.219 \mathrm{e}+09$

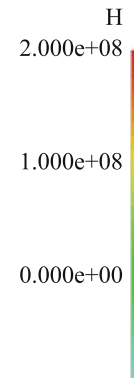

$-1.000 \mathrm{e}+08$

$-2.000 \mathrm{e}+08$ (b)

$\mathrm{E}[\mathrm{Y}]$

$6.925 \mathrm{e}+08$

$3.422 \mathrm{e}+08$

$-7.983 e+06$

$-3.582 \mathrm{e}+08$

$-7.084 \mathrm{e}+08$

(d)

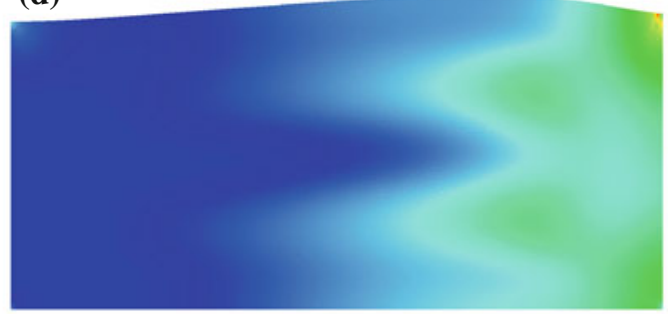

LAGRANGE

$-5.000 \mathrm{e}+19$

$-6.250 e+19$

$-7.500 e+19$

$-8.750 e+19$

$-1.000 \mathrm{e}+20$

Fig. 6 Electric $(\boldsymbol{e})$, magnetic $(\boldsymbol{h})$ and Lagrange multiplier $(\lambda)$ fields for $t=3.0 \times 10^{-8} \mathrm{~s}$ (not magnified)

ratio between the electromagnetic wave speed and the bulk wave speed $\sqrt{\kappa / \rho}$, which corresponds to the ratio between electrical and mechanical quantities in (41) is approximately 3500. Therefore, the problem is well conditioned.

\section{Discretization and time integration}

We use a variant of the MINI element by Arnold (cf. [29]) which was previously used for the Stokes equations to deal
Fig. 7 Corner crack: relevant data

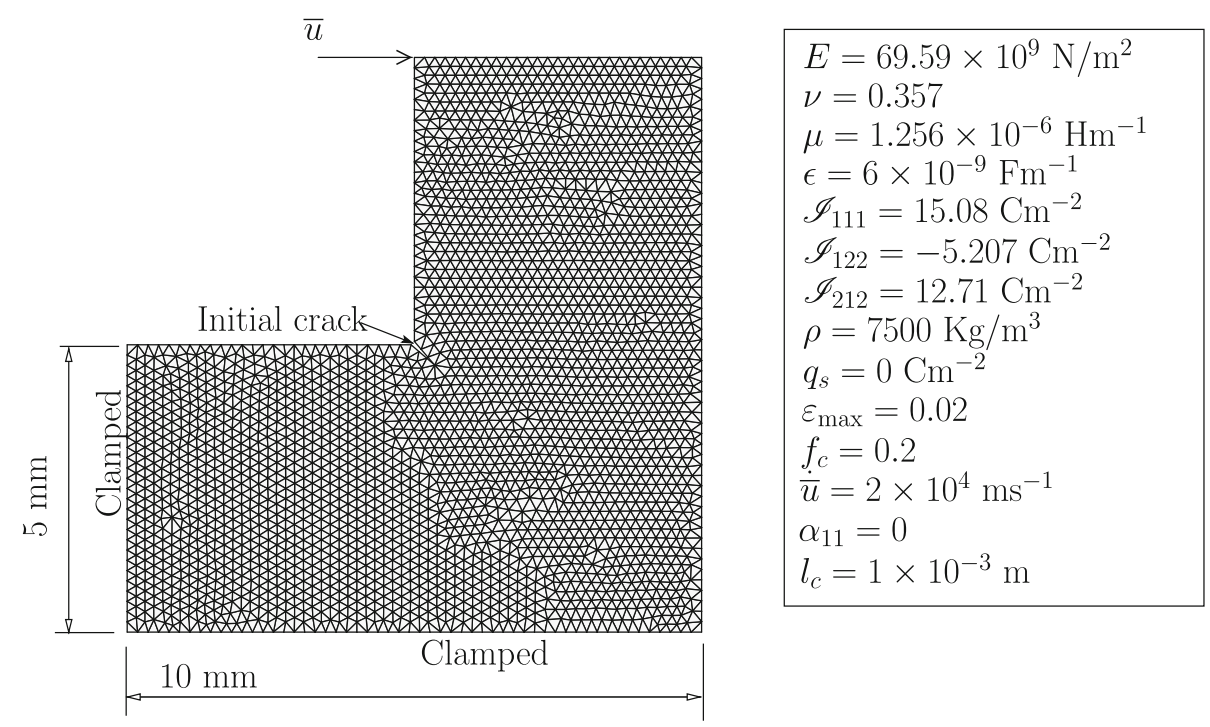


Fig. 8 Displacement/reaction results for the corner crack problem, comparing the three meshes. $1 \mathrm{~m}$ depth is considered

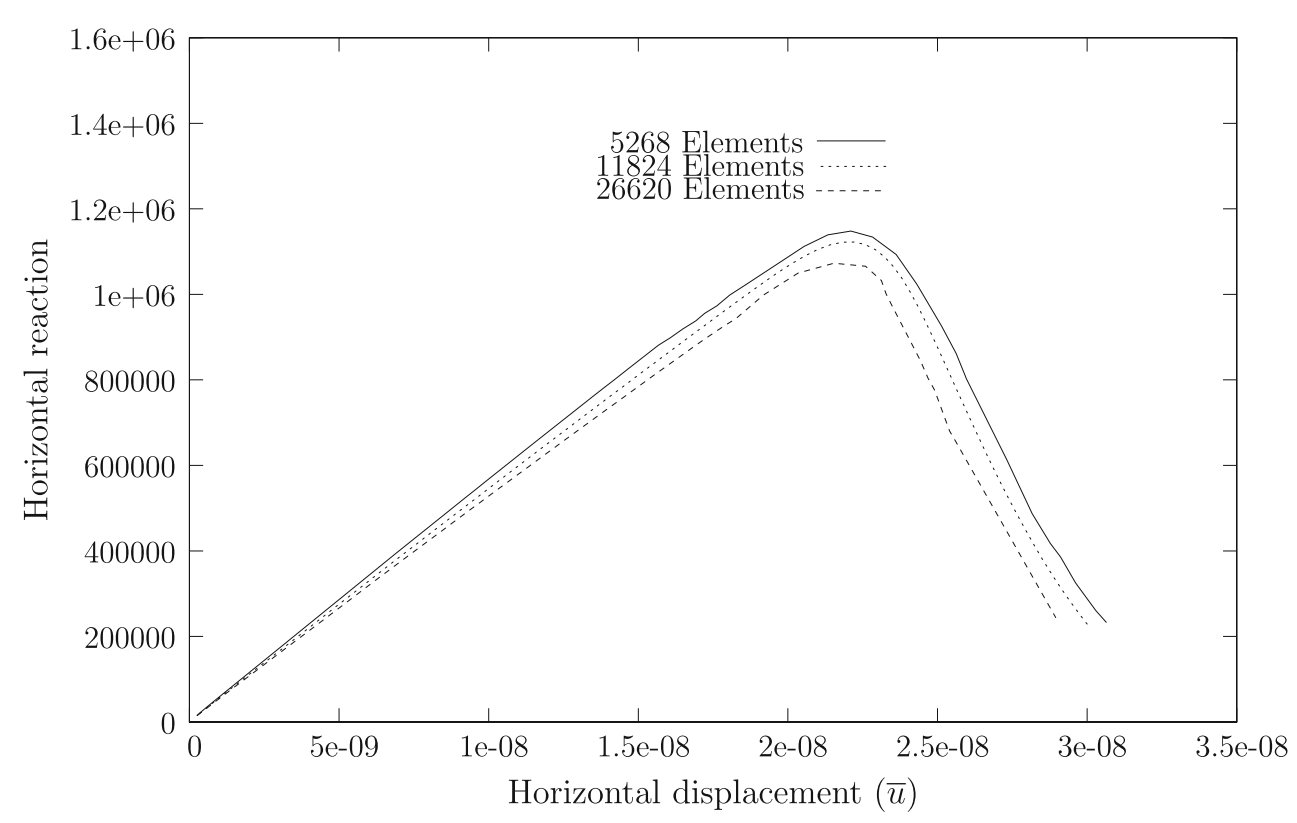

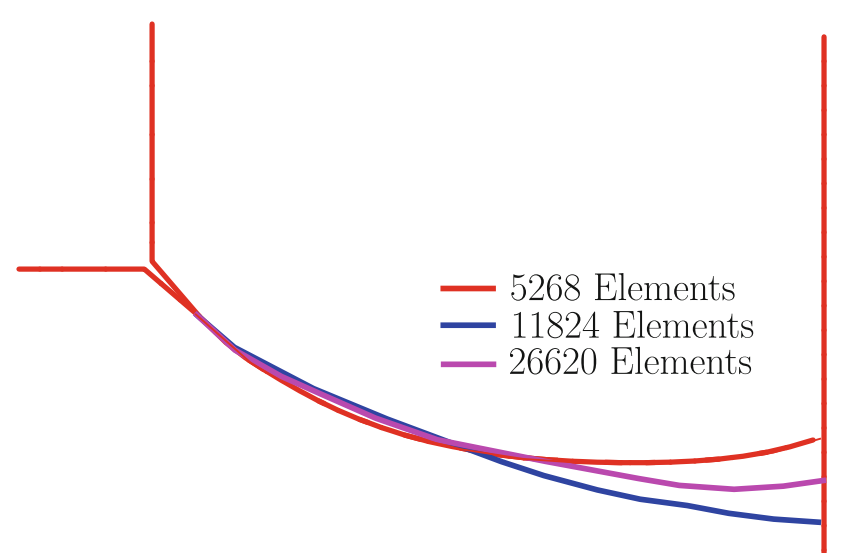

Fig. 9 Crack path comparison between the three meshes

with the mixed problem (unknowns $\boldsymbol{u}-\boldsymbol{e}-\boldsymbol{\lambda}$ ). We also used it before for finite strain plasticity. Figure 3 shows the element degrees-of-freedom and the corresponding shape functions.

Time integration follows the backward-Euler method for first-order time integration applied both to degrees-offreedom and their time-derivatives. If two consecutive timesteps are considered ( $n$ and $n+1$ ), the following expression results for $\ddot{\boldsymbol{a}}_{n+1}$ :

$\ddot{\boldsymbol{a}}_{n+1}=\frac{\boldsymbol{a}_{n+1}-\boldsymbol{a}_{n}}{\Delta t^{2}}-\frac{\dot{\boldsymbol{a}}_{n}}{\Delta t}$ where $\boldsymbol{a}$ contains degrees-of-freedom of all types $(\boldsymbol{u}, \boldsymbol{e}$ and $\lambda$ ). The velocity is approximated as $\dot{\boldsymbol{a}}_{n+1}=\left(\boldsymbol{a}_{n+1}-\boldsymbol{a}_{n}\right) / \Delta t$. With the aforementioned assumptions, the magnetic field at step $n+1$ can therefore be obtained as:

$\boldsymbol{b}_{n+1}=\boldsymbol{b}_{n}-\Delta t \nabla \times \boldsymbol{e}_{n+1}$

The magnetic field (43) is of course necessary for the boundary conditions and constitutive law and must be stored as an history variable. The relevant interpolated fields (with $h$ as the mesh characteristic length) in each element are obtained simply as:

$\begin{aligned} \boldsymbol{u}^{h} & =\sum_{K=1}^{3} N_{K}(\boldsymbol{\xi}) \boldsymbol{u}_{K} \\ \boldsymbol{e}^{h} & =\sum_{K=1}^{4} N_{K}(\boldsymbol{\xi}) \boldsymbol{e}_{K}\end{aligned}$

$\lambda^{h}=\sum_{K=1}^{3} N_{K}(\xi) \lambda_{K}$

where $N_{K}(\xi)$ are the shape functions represented in Fig. 3. After time integration, these are the only degrees-of-freedom required to solve the coupled problem since $\boldsymbol{b}$ is written as a function of $\boldsymbol{e}$. We keep track of the previous step $\left(\boldsymbol{a}_{n}\right.$ and $\dot{\boldsymbol{a}}_{n}$ ) which is sufficient to calculate $\ddot{\boldsymbol{a}}_{n}$ at the element level. The actual finite element implementation is too complex to be correctly and efficiently hand-coded and we resort to the Acegen software by Korelc ([27]) to accomplish this undertaking. The tip remeshing algorithm [30] is used, with the electric field boundary conditions at the crack faces reproducing the remaining boundary. 
(a)

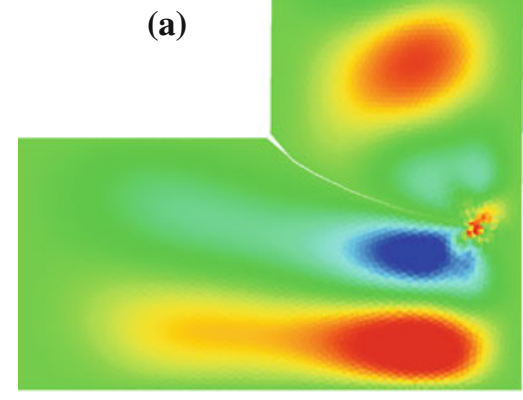

(c)

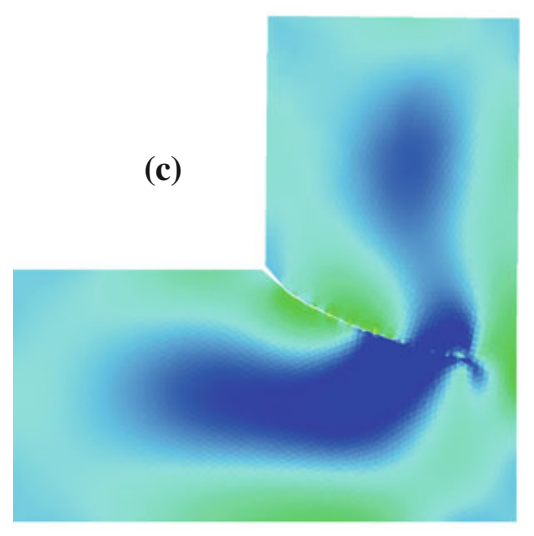

(e)
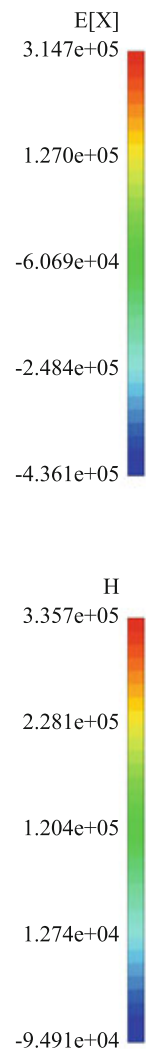

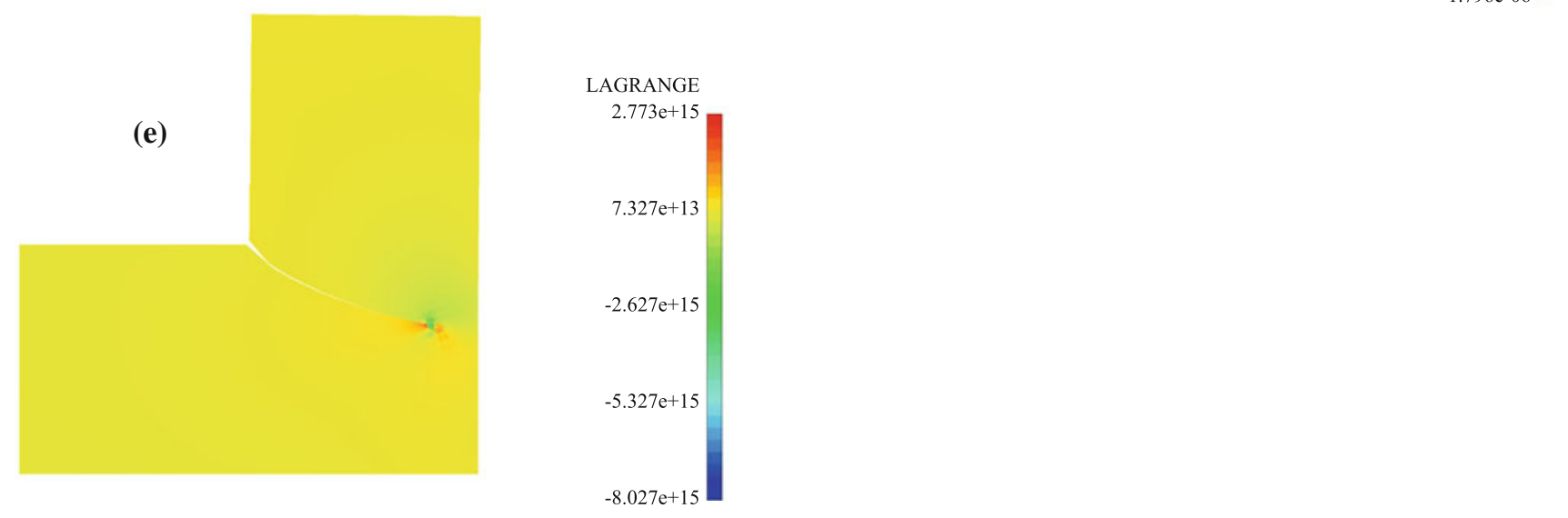

(b)

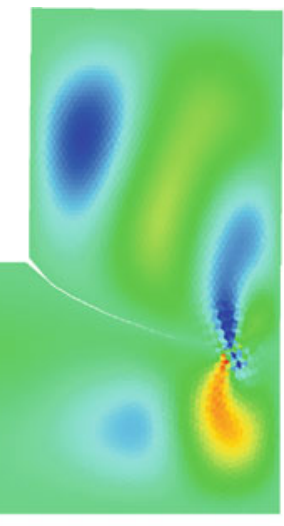

$\mathrm{E}[\mathrm{Y}]$ $5.243 \mathrm{e}+05$

$3.107 \mathrm{e}+05$

$9.722 \mathrm{e}+04$

$-1.163 e+05$

$-3.298 \mathrm{e}+05$

(d)

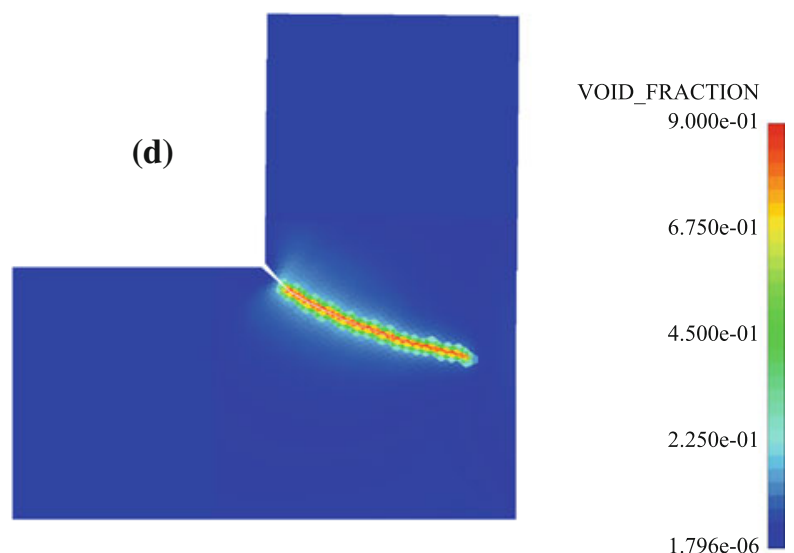

$1.796 \mathrm{e}-06$

Fig. 10 Electric $(\boldsymbol{e})$, magnetic $(\boldsymbol{h})$, Lagrange multiplier $(\lambda)$ and void fraction $(f)$ fields for $t=4.175 \times 10^{-8} \mathrm{~s}$. The mesh containing initially 11,824 elements is adopted. No displacement magnification is employed

\section{Numerical examples}

\subsection{Electric field test}

Surface electric charges ( $q_{s}$ in the above equations) cause electro-magnetic waves and, of course, stress waves (this is due to both the Maxwell stress term and the piezo-electric effect). Note that stress waves caused by the piezo-electric effect occur despite the absence of inertia $(\rho \ddot{\boldsymbol{u}}=\mathbf{0})$. With the purpose of obtaining a wave, we apply a time-constant electric charge at the surface of a straight bar and analyze the produced effect. Figure 4 shows the relevant quantities for this problem. A PZT material is considered, with properties shown in the same figure. Artificially large displacements are considered, with the goal of testing the robustness of implementation. Two meshes are tested: one containing 14,298 elements and 7,325 nodes and another with 25,374 elements and 12,922 nodes. The displacement at point (A) is shown in Fig. 5 for the two meshes. A slight difference is noticeable, a fact that does not occur in displacement-controlled problems. 
Fig. 11 Three-point bending test: relevant data and crack path results. A mesh with 6,585 nodes and with 12,858 triangles is employed
Fig. 12 Three-point bending test: vertical reaction as a function of $\bar{v}$
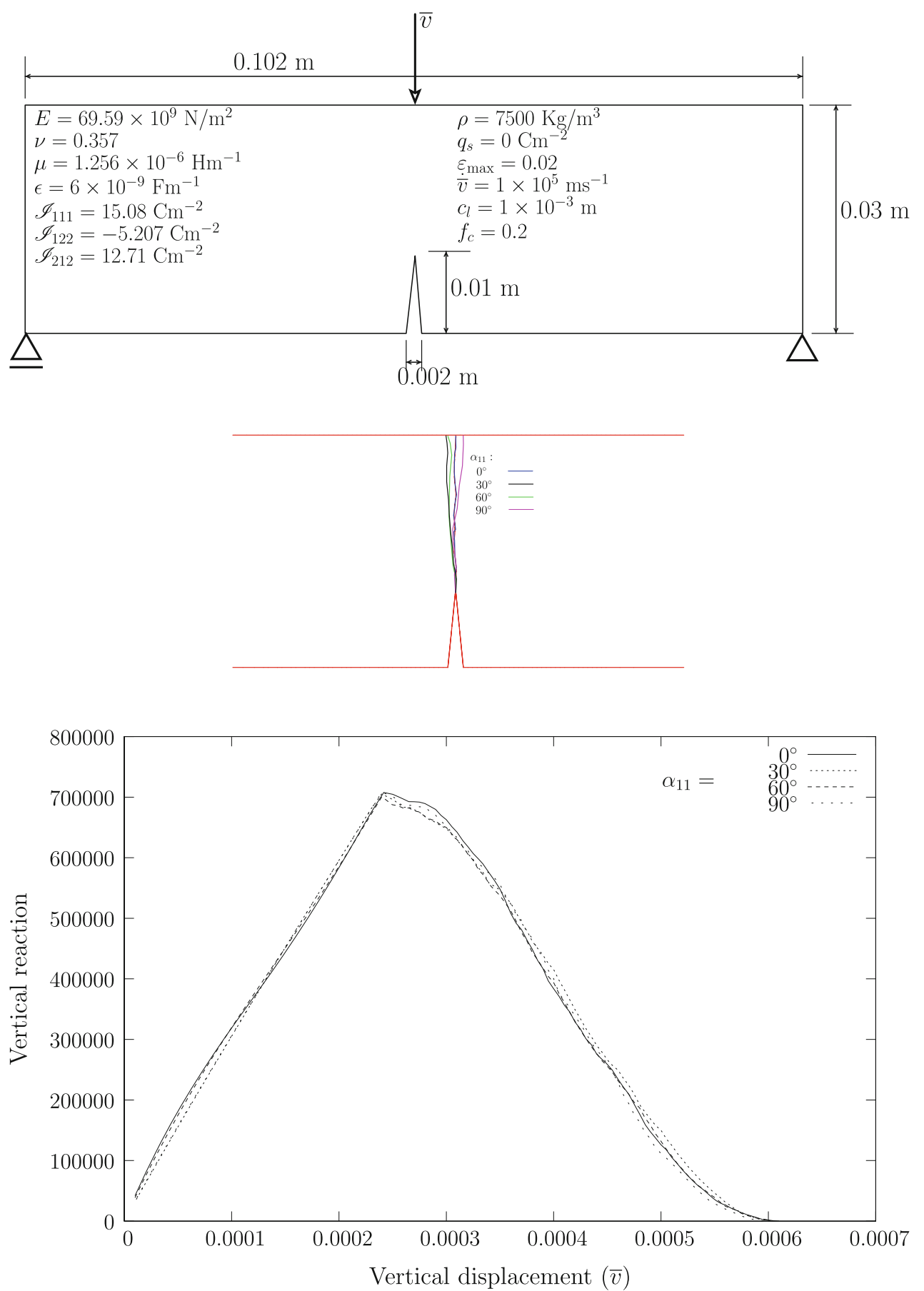

The contour plots of interested are shown in Fig. 6 for the finer mesh.

\subsection{Corner crack evolution}

The following problem is considered: a reentrant corner with a pre-existing $5 \mathrm{~mm}$ diagonal crack has two edges clamped and is subject to a constant velocity applied in a corner point.
Figure 7 shows the relevant data of this problem. Again, a PZT material is considered with typical properties (except $\varepsilon_{m}$ and $D_{c}$ which are introduced here for verification purposes, a fact not affecting the conclusions). Three uniformsized meshes are used to assert the sufficient independence of the results: 5268, 11824 and 26620 mixed triangular elements. The same time step of $\Delta t=2.5 \times 10^{-10} \mathrm{~s}$ is used for all meshes. Besides the mechanical boundary conditions depicted in Fig. 7 the electric field is constrained with 


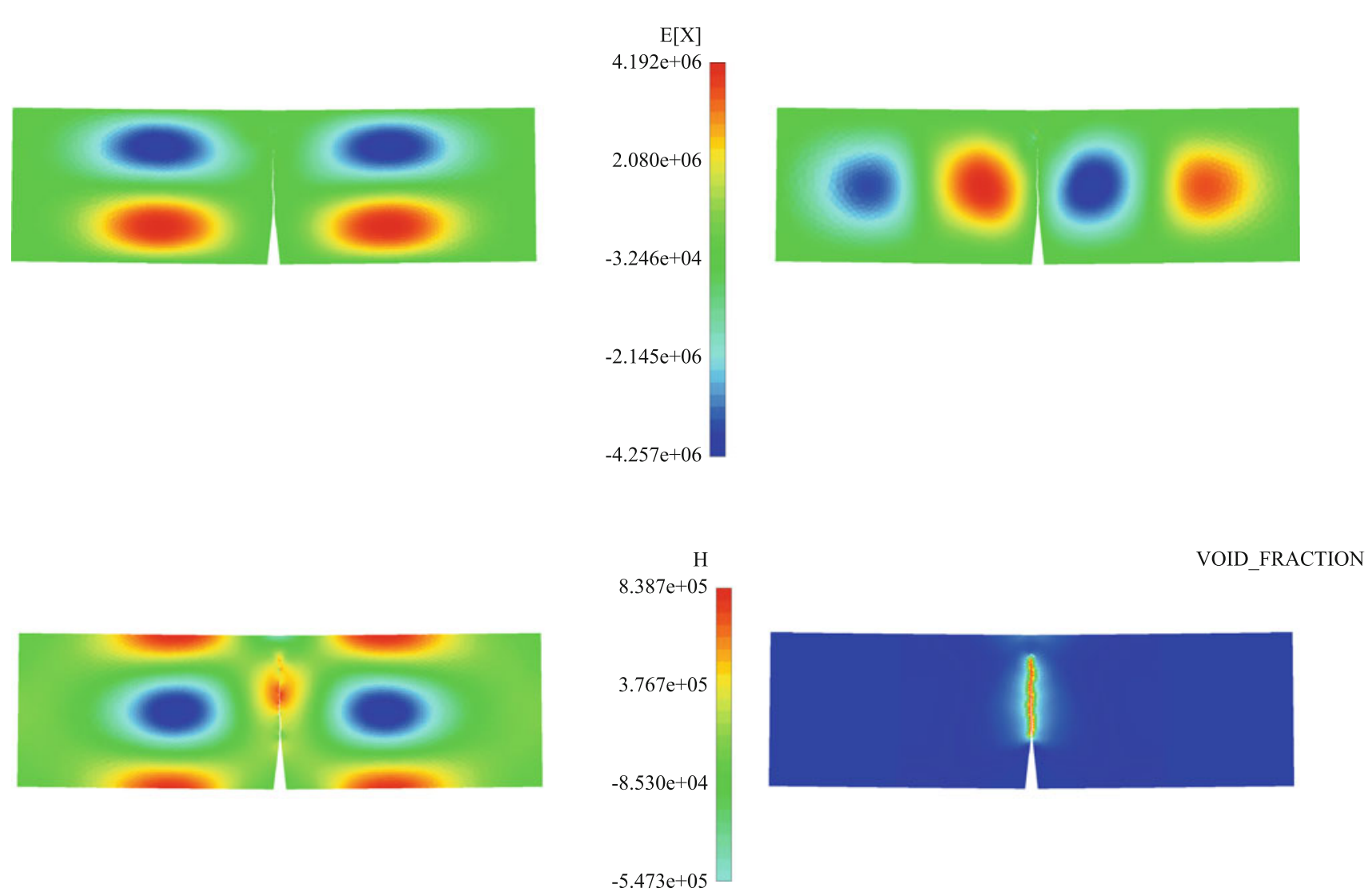

Fig. 13 Three-point bending test: electric $(\boldsymbol{e})$, magnetic $(\boldsymbol{h})$ and void fraction $(f)$ fields for $t=8.38 \times 10^{-9} \mathrm{~s}$ and $\alpha_{11}=0$

$q_{s}=0 \mathrm{Cm}^{-2}$. Now standard fracture algorithms (created by the first Author cf. [30-32]) are employed in this example with a insulating crack (see also [7]).

The reaction at the point of imposed displacement is monitored for the three meshes and the results are shown in Fig. 8. Close agreement occurs for the two finer meshes and very acceptable agreement is obtained in general. A comparison between the three crack paths is shown in Fig. 9 where adequate agreement can be observed. In addition, contour plots of relevant quantities for the finer mesh are shown in Fig. 10.

\subsection{Three-point bending test}

A version of the three-point bending beam is tested to assess the effect of the piezo-electric angle in the crack path curve and the load/displacement results. The relevant data for this problem is shown in Fig. 11 which also shows the crack paths for $\alpha_{11}=0, \pi / 6, \pi / 3$ and $\pi / 2$ radians. It is interesting to observe that, although we have imposed displacement, the piezo-electric effect affects the crack path. The reaction corresponding to $\bar{v}$ is less affected by the angle $\alpha_{11}$ as Fig. 12 illustrates. The relevant contour plots of $\boldsymbol{e}, \boldsymbol{h}$ and $f$ are shown in Fig. 13. It is also relevant to verify that the $y$-component of the electric field is anti-symmetric relative to a vertical plane, whereas other components follow geometrical symmetry. Of course there is some wave reflection near the null-charge boundaries.

\section{Discussion}

Specialized derivations both in the strong and weak forms of electro-magnetic coupling of dielectrics including piezoelectricity were presented. Crack propagation based on void fraction was introduced, which is new for electro-magnetic finite element models.

New finite element formulations in both the continuum element (with a variant of the MINI element) and the boundary condition element were shown and three verification numerical tests were presented. Compared with recent works on the same theme (e.g. [14]) the inclusion of the magnetic field, the derivation of novel electro-magnetic wave equations and the generalization of the boundary conditions were the major contributions. Further improvements in the number of ingredients and depth of numerical tests are being performed. The quality of implementation and corresponding results holds great promises for further generalization, in particular the adoption of consistent models for finite strains 
(as shown recently by Bustamante et al. [18] for the magnetic field). For imposed electric charge some mesh dependence was noted but is probably due to the basic time-integration algorithm.

Acknowledgments The authors gratefully acknowledge financing from the "Fundação para a Ciência e a Tecnologia" under the Project PTDC/EME-PME/108751 and the Program COMPETE FCOMP-010124-FEDER-010267. The first author is grateful to Professor Philipe Geubelle and Dr. Scot Breitenfeld (University of Illinois) for support of SIMPLAS ([33]). The second author acknowledges the support of "Fundação para a Ciência e a Tecnologia" for the grant SFRH/ BPD/63880/2009, and the "Fundação Calouste Gulbenkian" for "Estímulo à Criatividade e à Qualidade na Actividade de Investigação" in the Science Program of 2010.

\section{References}

1. Lax M, Nelson DF (1976) Maxwell equations in material form. Phys Rev B 13(4):1777-1784

2. Maugin GA (1988) Continuum mechanics of electromagnetic solids. Applied Mathematics and Mechanics, vol 33. NorthHolland, Amsterdam

3. Ericksen JL (2007) On formulating and assessing continuum theories of electromagnetic fields in elastic materials. J Elast 87:95-108

4. Ericksen JL (2007) Theory of elastic dielectrics revisited. Arch Ration Mech Anal 183:299-313

5. Dorfmann A, Ogden RW (2006) Nonlinear electroelastic deformations. J Elast 82(2):99-127

6. Belahcen A, Fonteyn K (2008) On numerical modeling of coupled magnetoelastic problem. In: Kvamsdal T, Mathisen KM, Pettersen B (eds) 21st nordic seminar on computational mechanics. NSCM, Barcelona, CIMNE

7. Kuna M (2010) Fracture mechanics of piezoelectric materialswhere are we right now. Eng Fract Mech 77:309-326

8. Kuna M (2006) Finite element analyses of cracks in piezoelectric structures: a survey. Arch Appl Mech 76:725-745

9. Bathe K-J (1996) Finite element procedures. Prentice-Hall, Englewood Cliffs

10. Ogden RW (1997) Nonlinear elastic deformations. Dover Publications, Mineola, NY

11. Marsden JE, Hughes TJR (1994) Mathematical foundations of elasticity. Dover Publications, New York

12. Haus HA, Melcher JR (1989) Electromagnetic fields and energy. Prentice-Hall, Englewood Cliffs

13. Jackson JD (1999) Classical electrodynamics, 3rd edn. Wiley, New York
14. Mota A, Zimmerman JA (2011) A variational, finite-deformation constitutive model for piezoelectric materials. Int J Numer Methods Eng 85:752-767

15. Bustamante R, Ogden RW (2006) Universal relations for nonlinear electroelastic solids. Acta Mech 182:125-140

16. Bustamante R, Dorfmann A, Ogden RW (2006) Universal relations in isotropic nonlinear magnetoelasticity. Q J Mech Appl Math 59(3):435-450

17. Linder C, Rosato D, Miehe C (2011) New finite elements with embedded strong discontinuities for the modeling of failure in electromechanical coupled solids. Comp Method Appl Mech Eng 200:141-161

18. Bustamante R, Dorfmann A, Ogden RW (2011) Numerical solution of finite geometry boundary-value problems in nonlinear magnetoelasticity. Int J Solids Struct 48:874-883

19. Bonet J, Wood RD (2008) Nonlinear continuum mechanics for finite element analysis, Second edition. Cambridge University Press, Cambridge

20. Vu DK, Steinmann P, Possart G (2007) Numerical modelling of non-linear electroelasticity. Int J Numer Methods Eng 70:685-704

21. Lemaitre J, Chaboche J-L (1990) Mechanics of solid materials. Cambridge University Press, Cambridge

22. Lemaitre J (1996) A course on damage mechanics, Second edition. Springer, Berlin

23. Mullins L (1969) Softening of rubber by deformation. Rubber Chem Technol 42:339-362

24. Oliver J (1989) A consistent characteristic length for smeared cracking models. Int J Numer Methods Eng 28:461-474

25. Truesdell C, Noll W (2004) The non-linear field theories of mechanics, Third edition. Springer, Berlin

26. Hughes TJR (2000) The finite element method. Linear static and dynamic finite element analysis. Dover Publications, New York (reprint of Prentice-Hall edition, 1987)

27. Korelc $\mathbf{J}$ (2002) Multi-language and multi-environment generation of nonlinear finite element codes. Eng Comput 18(4):312-327

28. Wolfram Research Inc. (2008) Mathematica, Version 7.0, Champaign, IL

29. Arnold DN, Brezzi F, Fortin M (1984) A stable finite element for the Stokes equations. Calcolo XXI(IV):337-344

30. Areias P, Dias-da-Costa D, Alfaiate J, Júlio E (2009) Arbitrary bi-dimensional finite strain cohesive crack propagation. Comput Mech 45(1):61-75

31. Areias P, Van Goethem N, Pires EB (2011) A damage model for ductile crack initiation and propagation. Comput Mech 47(6): 641-656

32. Areias P, Van Goethem N, Pires EB (2011) Constrained ale-based discrete fracture in shells with quasi-brittle and ductile materials. In: CFRAC 2011 international conference, Barcelona, Spain, June 2011. CIMNE

33. Areias P. Simplas. https://ssm7.ae.uiuc.edu:80/simplas. Accessed 15 June 2012 